\title{
The Crepant Resolution Conjecture
}

\author{
Jim Bryan and Tom Graber
}

\begin{abstract}
For orbifolds admitting a crepant resolution and satisfying a hard Lefschetz condition, we formulate a conjectural equivalence between the GromovWitten theories of the orbifold and the resolution. We prove the conjecture for the equivariant Gromov-Witten theories of $\mathrm{Sym}^{n} \mathbb{C}^{2}$ and $\mathrm{Hilb}^{n} \mathbb{C}^{2}$.
\end{abstract}

\section{Introduction}

1.1. Overview. Gromov-Witten theory is a mathematical counterpart of topological string theory in physics. A well known principle in physics states that string theory on an orbifold is equivalent to string theory on a crepant resolution $[\mathbf{3 8}, \mathbf{4 2}]$. In their ground breaking paper [15], Chen and Ruan define orbifold quantum cohomology using an orbifold version of Gromov-Witten theory. Orbifold GromovWitten theory was developed in the algebro-geometric context in $[1,3,2]$ using Abramovich and Vistoli's notion of twisted stable maps to a Deligne-Mumford stack.

The Gromov-Witten invariants of a projective manifold $Y$ are multilinear functions $\langle\cdots\rangle_{g, \beta}^{Y}$ on its cohomology $H^{*}(Y)$. The Gromov-Witten invariants of a orbifold $\mathcal{X}$ are multilinear functions $\langle\cdots\rangle_{g, \beta}^{\mathcal{X}}$ on the orbifold cohomology $H_{\text {orb }}^{*}(\mathcal{X})$. Orbifold cohomology is by definition the ordinary cohomology of the inertia stack $I \mathcal{X}$ with a shifted grading $[\mathbf{2}, \mathbf{1 5}] . H_{\text {orb }}^{*}(\mathcal{X})$ contains the usual cohomology $H^{*}(X)$ as a subspace and its orthogonal complement is referred to as the space of twisted sectors. If $\mathcal{X}$ is a Gorenstein orbifold whose coarse moduli scheme $X$ admits a crepant resolution $Y \rightarrow X$, Yasuda has proven that $H_{\text {orb }}^{*}(\mathcal{X}, \mathbb{C})$ and $H^{*}(Y, \mathbb{C})$ are isomorphic as graded vector spaces [41]. Yasuda's proof provides an equality of Betti numbers but does not provide any natural choice of isomorphism. Nevertheless, it has been suggested by Ruan [36] that there should be such an isomorphism which identifies the Gromov-Witten theories. He proposes that specializing the values of certain quantum parameters of the small quantum cohomology of the resolution will recover the orbifold cohomology of the orbifold. In this paper, we formulate an analogous conjecture at the level of the full genus zero quantum potentials, and we explore its consequences. In particular, we show this conjecture allows one to essentially recover the Gromov-Witten theory of the resolution in terms of that of the orbifold. We confirm the validity of our conjecture for some examples including the case of $\mathcal{X}=\operatorname{Sym}^{n} \mathbb{C}^{2}, Y=\operatorname{Hilb}^{n} \mathbb{C}^{2}$. 
Recent work of Coates, Corti, Iritani, and Tseng, [19] strongly suggests that for orbifolds failing the hard Lefschetz condition, the relationship between the GromovWitten theories of the orbifold and its resolution is more complicated and is better expressed in the more sophisticated framework of Givental's Lagrangian formalism. We are grateful to them for bringing the hard Lefschetz condition to our attention.

1.2. Notation. By an orbifold, we will mean a smooth algebraic DeligneMumford stack over $\mathbb{C}$. An orbifold $\mathcal{X}$ is said to be Gorenstein if $\mathcal{X}$ has generically trivial stabilizers and the canonical bundle of $\mathcal{X}$ pulls back from a line bundle on the coarse moduli space $X$ (equivalently, for every $x \in \mathcal{X}$, the action of the isotropy group on the canonical line bundle is trivial). A resolution of singularities $\pi: Y \rightarrow X$ is called crepant if $K_{Y}=\pi^{*} K_{X}$.

Let $\mathcal{X}$ be a Gorenstein orbifold and let

$$
\pi: Y \rightarrow X
$$

be a crepant resolution of the coarse moduli space $X$. We say that an integer basis for the second homology group of a variety is positive if the cone generated by the basis contains the Mori cone.

Let $\left\{\beta_{1}, \ldots, \beta_{r}\right\}$ be a positive basis of $H_{2}(Y)$ such that $\left\{\beta_{s+1}, \ldots, \beta_{r}\right\}$ is a basis for the kernel of $\pi_{*}: H_{2}(Y) \rightarrow H_{2}(X)$. Note that $\left\{\pi_{*} \beta_{1}, \ldots, \pi_{*} \beta_{s}\right\}$ is a positive basis for $H_{2}(X)$. We choose a basis $\left\{\gamma_{0}, \ldots, \gamma_{a}\right\}$ for $H^{*}(Y)$, with $\gamma_{0}=1$ and $\gamma_{1}, \ldots, \gamma_{r}$ the basis for $H^{2}(Y)$ dual to the $\beta_{i}$.

The genus zero Gromov-Witten invariants of $Y$ are multilinear functions $\langle\cdots\rangle_{\beta}^{Y}$ on $H^{*}(Y)$, defined by cohomological evaluations against $\left[\bar{M}_{0, n}(Y, \beta)\right]^{\text {vir }}$, the virtual fundamental class of the moduli space of stable maps $[21,27]$. The invariants are encoded in the potential function,

$$
F^{Y}\left(y_{0}, \ldots, y_{a}, q_{1}, \ldots, q_{r}\right)=\sum_{n_{0}, \ldots, n_{a}=0}^{\infty} \sum_{\beta}\left\langle\gamma_{0}^{n_{0}} \cdots \gamma_{a}^{n_{a}}\right\rangle_{\beta}^{Y} \frac{y_{0}^{n_{0}}}{n_{0} !} \cdots \frac{y_{a}^{n_{a}}}{n_{a} !} q_{1}^{d_{1}} \cdots q_{T}^{d_{r}}
$$

where $\beta=d_{1} \beta_{1}+\cdots+d_{r} \beta_{r}$ is summed over all $r$-tuples $\left(d_{1}, \ldots, d_{r}\right)$ of non-negative integers.

Similarly, the genus zero Gromov-Witten invariants of $\mathcal{X}$ are multilinear functions $\langle\cdots\rangle_{\beta}^{\mathcal{X}}$ on $H_{o r b}^{*}(\mathcal{X})$, defined by cohomological evaluations against the virtual fundamental class $\left[\bar{M}_{0, n}(\mathcal{X}, \beta)\right]^{\mathrm{vir}}$ of the moduli space of twisted stable maps [2]. We choose a basis $\left\{\delta_{0}, \ldots, \delta_{a}\right\}$ for $H_{\text {orb }}^{*}(\mathcal{X})$ and we define the potential function for $\mathcal{X}$ :

$$
F^{\mathcal{X}}\left(x_{0}, \ldots, x_{a}, u_{1}, \ldots, u_{s}\right)=\sum_{n_{0}, \ldots, n_{a}=0}^{\infty} \sum_{\beta}\left\langle\delta_{0}^{n_{0}} \cdots \delta_{a}^{n_{a}}\right\rangle_{\beta}^{\mathcal{X}} \frac{x_{0}^{n_{0}}}{n_{0} !} \cdots \frac{x_{a}^{n_{a}}}{n_{a} !} u_{1}^{d_{1}} \cdots u_{s}^{d_{s}}
$$

where $\beta=d_{1} \pi_{*} \beta_{1}+\cdots+d_{s} \pi_{*} \beta_{s}$ is summed over all $s$-tuple $\left(d_{1}, \ldots, d_{s}\right)$ of nonnegative integers.

The inertia stack $I \mathcal{X}$ of an orbifold $\mathcal{X}$ is defined to be the fibered product of $\mathcal{X}$ with itself over the diagonal in $\mathcal{X} \times \mathcal{X}$. The points of $I \mathcal{X}$ are pairs $(x, g)$ where $x \in \mathcal{X}$ and $g \in \operatorname{Aut}_{\mathcal{X}}(x)$. There is an involution $I$ of $I \mathcal{X}$ taking $(x, g)$ to $\left(x, g^{-1}\right)$. To each component $\mathcal{X}_{i}$ of $I \mathcal{X}$ we assign a rational number age $\left(\mathcal{X}_{i}\right)$ as follows. Let $(x, g)$ be a point in $\mathcal{X}_{i}$. Then $g$ acts on $T_{x} \mathcal{X}$ with eigenvalues $\left(\alpha_{1}, \ldots, \alpha_{n}\right)$ where $n=\operatorname{dim} \mathcal{X}$. Let $o(g)$ be the order of $g$ and define $s_{j} \in 0, \ldots, o(g)-1$ by $\alpha_{j}=\exp \left(2 \pi i \frac{s_{j}}{o(g)}\right)$. 
Then age is defined by

$$
\operatorname{age}\left(\mathcal{X}_{i}\right)=\frac{1}{o(g)} \sum_{j=1}^{n} s_{j}
$$

Age is well defined and is integral for Gorenstein orbifolds.

As a graded vector space, the orbifold cohomology of $\mathcal{X}$ is the cohomology of $I \mathcal{X}$ with the grading shifted by twice the age:

$$
H_{\text {orb }}^{*}(\mathcal{X})=\bigoplus_{\mathcal{X}_{i} \subset I \mathcal{X}} H^{*-2 \operatorname{age}\left(\mathcal{X}_{i}\right)}\left(\mathcal{X}_{i}\right)
$$

Suppose that the coarse moduli space $X$ is projective with hyperplane class $\omega$. In [24], Fernandez asked if the hard Lefschetz isomorphism holds in orbifold cohomology, namely if the operator $L_{\omega}$ given by multiplication by $\omega$ in the orbifold cohomology ring, induces isomorphisms

$$
L_{\omega}^{p}: H_{o r b}^{n-p}(\mathcal{X}) \rightarrow H_{o r b}^{n+p}(\mathcal{X})
$$

Fernandez proved that $L_{\omega}^{p}$ is an isomorphism for all $\omega$ if and only if the age is invariant under the involution $I$. We call this condition (also defined for nonprojective orbifolds) the hard Lefschetz condition.

DEFINITION 1.1. An orbifold $\mathcal{X}$ is said to satisfy the hard Lefschetz condition if the involution

$$
I: I \mathcal{X} \rightarrow I \mathcal{X}
$$

preserves the age.

Note that this condition is satisfied by holomorphic symplectic orbifolds.

1.3. The Conjecture. Our main conjecture relates the two potential functions $F^{Y}$ and $F^{\mathcal{X}}$.

Conjecture 1.2 (Crepant Resolution Conjecture). Given an orbifold $\mathcal{X}$ satisfying the hard Lefschetz condition and admitting a crepant resolution $Y$, there exists a graded linear isomorphism

$$
L: H_{\text {orb }}^{*}(\mathcal{X}) \rightarrow H^{*}(Y)
$$

and roots of unity $c_{s+1}, \ldots, c_{r}$ such that the following conditions hold.

(1) $L$ extends the map $\pi^{*}: H^{*}(\mathcal{X}) \rightarrow H^{*}(Y)$.

(2) Regarding the potential function $F^{Y}$ as a power series in the parameters $y_{0}, \ldots, y_{a}, q_{1}, \ldots, q_{s}$, the coefficients admit analytic continuations from $\left(q_{s+1}, \ldots, q_{r}\right)=(0, \ldots, 0)$ to $\left(q_{s+1}, \ldots, q_{r}\right)=\left(c_{s+1}, \ldots, c_{r}\right)$. 
(3) The potential functions $F^{\mathcal{X}}$ and $F^{Y}$ are equal after the substitution ${ }^{1}$

$$
\begin{aligned}
y_{i} & =\sum_{j} L_{i}^{j} x_{j} \\
q_{i} & = \begin{cases}c_{i} & \text { when } i>s \\
u_{i} & \text { when } i \leq s .\end{cases}
\end{aligned}
$$

REMARK 1.3. The cohomological parameters which appear in the potential functions, $\left\{x_{0}, \ldots x_{a}\right\}$ and $\left\{y_{0}, \ldots, y_{a}\right\}$ are equal in number by Yasuda's result. However, the number of quantum parameters, $\left\{u_{1}, \ldots, u_{s}\right\}$ and $\left\{q_{1}, \ldots, q_{r}\right\}$ differ, and so naïvely, the potential function $F^{Y}$ appears to have more information than $F^{\mathcal{X}}$. However, the divisor equation implies that the potential function $F^{Y}$ contains redundant information. In fact, given $L, c_{s+1}, \ldots, c_{r}$, and $F^{\mathcal{X}}$, one can essentially recover $F^{Y}$. This will be made more clear in section 2 , where we will present an alternative but equivalent formulation of the conjecture which is particularly convenient when studying the small quantum cohomology ring. Rather than resolving the difference in the number of $q$ 's and $u$ 's by setting some of the $q$ 's to constants, one can adjoin extra $u$ variables to the orbifold partition function, by defining a generalized notion of degree for orbifold curves with unmarked twisted points.

REMARK 1.4. A finite set of coefficients in these potential functions are not well defined since certain degenerate moduli spaces do not exist. Namely, terms of degree zero in the quantum parameters and of degree less than three in the cohomological variables are undefined. We are not making any conjectures about these coefficients. To get a precise equality, one needs to either take triple derivatives of the series on both sides, or choose compatible assignments of values to the unstable coefficients. It would be interesting to find a meaningful way of defining these unstable invariants.

REMARK 1.5. It is a consequence of the conjecture that the linear map $L$ must preserve the (orbifold) Poincaré pairing.

REMARK 1.6. If $\mathcal{X}$ admits an action of an algebraic torus $T$ and $Y$ is a $T$ equivariant crepant resolution, then we can extend the conjecture to include equivariant parameters. In fact, this equivariant version of the conjecture follows from the absolute version, by considering the conjecture applied to finite dimensional approximations to the homotopy quotients $Y_{T} \rightarrow X_{T}$.

REMARK 1.7. The coefficients of $F^{Y}$ and $F^{\mathcal{X}}$ are rational numbers, but in general the linear transformation $L$ may be have to be defined over some extension of $\mathbb{Q}$. A consequence of the conjecture is that there is a symmetry of $F^{Y}$ given by the action of the Galois group of the extension on the change of variables. In practice, this is often a highly non-trivial symmetry. For an example, see remark 3.12 .

\footnotetext{
${ }^{1}$ Update, April, 2008: It is now known that this condition is too strong; it should be weakened to $q_{i}=c_{i} u_{i}$ in the case where $i \leq s$ where $c_{i}$ are constants. There has been significant progress on the crepant resolution conjecture since this paper was written. Many more examples have been understood $[4,6,8,9,10,14,16,17,19,25,28,40]$, and significant conceptual progress has been made $[18,20]$. The paper [20] provides the most general version of the conjecture, valid without the hard Lefscetz condition and applicable in all genera. In $[20, \S 9]$, the general conjecture is shown to reduce to our conjecture, with the aforementioned weakening. This weaking is needed as is shown by the example of Gillam [25].
} 
REMARK 1.8. Our conjecture should also hold as stated for higher genus potentials. There is very little evidence in positive genus, although Maulik's computation of the full Gromov-Witten potential for $A_{n}$ surface resolution [31] does provide some positive evidence.

1.4. The noncompact case. Although Gromov-Witten theory is best known in the compact setting, the simple examples we want to focus on are non-compact, so we observe that there is a large class of noncompact examples where there is a well defined version of the conjecture.

The most convenient hypothesis here is to assume that $X$ is projective over an affine scheme and that $Y$ is projective over $X$ and hence also projective over an affine. (In fact, in our examples $X$ will actually be affine.) In this setting, although the spaces of stable (twisted) maps need not be proper, the evaluation maps from the space of maps to $Y$ (or $I \mathcal{X}$ ) will be proper. Thus we have well-defined GromovWitten classes

$$
\left\langle\gamma_{1}, \ldots, \gamma_{n}, *\right\rangle_{\beta}
$$

defined as in [1] by pushing forward from the space of $n+1$ pointed (twisted) stable maps to $Y$ (or $I \mathcal{X})$.

If the target is projective, then because of the formula

$$
\left\langle\gamma_{1}, \ldots, \gamma_{n+1}\right\rangle_{\beta}=\gamma_{n+1}\left(\left\langle\gamma_{1}, \ldots, \gamma_{n}, *\right\rangle_{\beta}\right)
$$

these homology valued invariants contain equivalent information to the numerical Gromov-Witten invariants, and moreover, the conjecture as stated implies immediately a conjecture for a generating series of homology valued invariants. In the noncompact setting, where one cannot reduce the homology classes to numbers in this way, we can then use these invariants to make a meaningful version of the conjecture.

In fact, we will not need to pursue a careful language for these refined invariants, since our examples have another useful feature. They all admit a torus action with compact fixed locus. Because of this, there is a perfect pairing on the localized $T$-equivariant cohomology given by formally applying the Bott residue formula. While this pairing takes values in $H_{T}^{*}(p t, \mathbb{C})_{\mathfrak{m}}$, rather than $\mathbb{C}$, it still allows us to do calculations at the level of the familiar generating functions for numerical Gromov-Witten invariants with the slight novelty that some of these numbers will be rational functions in the equivariant parameters.

\section{Degree in twisted sectors and Quantum cohomology}

In this section we extend the definition of $\langle\cdots\rangle_{g, \beta}^{\mathcal{X}}$ to allow for $\beta$ to be a "curve class" in the twisted sector. Consequently, the corresponding Gromov-Witten potential of $\mathcal{X}$ includes quantum parameters corresponding to twisted sectors. This allows us to formulate an alternative version of the Crepant Resolution Conjecture where the number of variables for $\mathcal{X}$ and for $Y$ are the same. In particular, the large and small quantum cohomology rings of $Y$ and $\mathcal{X}$ have the same number of deformation parameters and are isomorphic (in a certain sense - see subsection 2.3) when the Crepant Resolution Conjecture holds.

2.1. The orbifold Neron-Severi group and twisted degrees. We define an enlarged Neron-Severi group for a Gorenstein orbifold $\mathcal{X}$ as follows. Let $T^{1}(\mathcal{X})$ be the twisted part of $H_{\text {orb }}^{2}(\mathcal{X}, \mathbb{Z})$. As this is generated by fundamental classes 
of certain irreducible components of the inertia stack, it comes with a canonical (unordered) basis and is a free Abelian group of rank $r-s$.

DeFinition 2.1. We define the orbifold Neron-Severi group $\widehat{N S}_{1}(\mathcal{X})$ by

$$
\widehat{N S}_{1}(\mathcal{X})=N S_{1}(\mathcal{X}) \oplus T^{1}(\mathcal{X})^{\vee}
$$

That is, an element $\widehat{\beta} \in \widehat{N S}_{1}(\mathcal{X})$ is a curve class $\beta$ in $\mathcal{X}$ together with a function $\widehat{\beta}(i)$ assigning an integer to each age one component of $I \mathcal{X}$. An element of $\widehat{N S}_{1}(\mathcal{X})$ will be considered effective if the underlying curve class is effective, and the function is nonnegative.

Recall that evaluation at the $i$ th point of a twisted stable map takes values in the inertia stack and defines a virtual morphism $e_{i}: \bar{M}_{g, n}(\mathcal{X}, \beta) \rightarrow I \mathcal{X}$. (We are using different conventions here than those of [2] or [1] - our $\bar{M}$ corresponds to $\mathcal{K}$ and our $e_{i}$ corresponds to $\tilde{e}_{i}$ of Proposition 6.1 .4 of $[1]$.)

DeFinition 2.2. Given an effective class $\hat{\beta} \in \widehat{N S}_{1}(\mathcal{X})$, we define $\bar{M}_{g, n}(\mathcal{X}, \hat{\beta})$ to be the moduli space parameterizing genus $g$ twisted stable maps to $\mathcal{X}$ with degree $\beta$ with $n$ ordered marked points and with $\hat{\beta}(i)$ unordered twisted points which map to $D_{i}$, the ith component of the inertia stack. Precisely, if we consider the following fiber product:

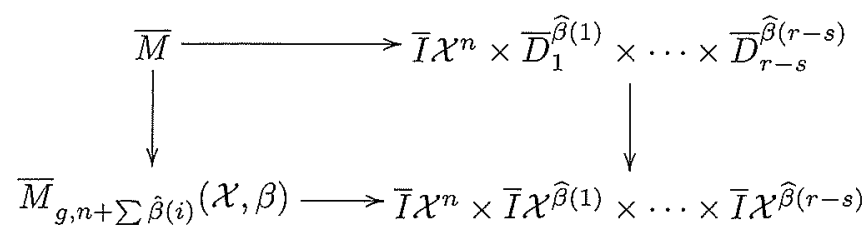

then we define $\bar{M}_{g, n}(\mathcal{X}, \widehat{\beta})$ to be the quotient $\left[\bar{M} / S_{\widehat{\beta}(1)} \times \cdots \times S_{\widehat{\beta}(r-s)}\right]$. Here $\bar{I} \mathcal{X}$ is the rigidified stack and $\bar{D}_{i}$ is the ith component.

REMARK 2.3. One interpretation of the usual degree is as counting the number of times a curve intersects some fixed divisor. Similarly, we can interpret the degree in the twisted sector as counting the number of times some curve "intersects" some twisted divisor, namely it gives the number of (unmarked, non-nodal) stacky points that get mapped to the corresponding age one component of the inertia stack. The reason for not including nodal stacky points in the count is so that the degree will be locally constant in families. We ignore the marked points so that degree is additive when gluing smooth curves together to form nodal ones, and therefore the boundary of the moduli spaces $\bar{M}_{g, n}(\mathcal{X}, \widehat{\beta})$ have a product description analogous to the usual one for the ordinary stable map moduli spaces.

2.2. Gromov-Witten invariants for degrees in twisted sectors and the divisor equation. Since the right hand vertical arrow of the diagram in Definition 2.2 is simply an inclusion of a union of connected components, so is the left hand vertical arrow, which means that the perfect obstruction theory and virtual fundamental class for the usual space of twisted stable maps immediately give one on $\bar{M}$, and by descent, we get a virtual fundamental class on $\bar{M}_{g, n}(\mathcal{X}, \widehat{\beta})$.

We can thus use these moduli spaces to define extended Gromov-Witten invariants which include degrees in the twisted sectors. Correspondingly, we define the 
genus zero extended Gromov-Witten potential of $\mathcal{X}$ by

$$
\begin{aligned}
& \widehat{F}^{\mathcal{X}}\left(x_{0}, \ldots, x_{a}, u_{1}, \ldots, u_{r}\right)= \\
& \left.\qquad \sum_{n_{0}, \ldots, n_{a}=0}^{\infty} \sum_{\widehat{\beta}}\left\langle\delta_{0}^{n_{0}} \cdots \delta_{a}^{n_{k}}\right\rangle\right\rangle_{\widehat{\beta}}^{\mathcal{X}} \frac{x_{0}^{n_{0}}}{n_{0} !} \cdots \frac{x_{a}^{n_{a}}}{n_{a} !} u_{1}^{d_{1}} \cdots u_{s}^{d_{s}} u_{s+1}^{\widehat{\beta}(1)} \cdots u_{r}^{\widehat{\beta}(r-s)}
\end{aligned}
$$

The extended invariants do not contain any new information, since we have the following obvious formula:

$$
\left\langle D_{1}^{\widehat{\beta}(1)} \cdots D_{r-s}^{\widehat{\beta}(r-s)} \alpha_{1} \cdots \alpha_{n}\right\rangle_{\beta}^{\mathcal{X}}=\widehat{\beta}(1) ! \cdots \widehat{\beta}(r-s) ! \cdot\left\langle\alpha_{1} \cdots \alpha_{n}\right\rangle_{\widehat{\beta}}^{\mathcal{X}}
$$

which immediately reduces the calculation of these "new" invariants to the calculation of the standard orbifold invariants. We think of this as the analog of the divisor equation for the "twisted divisors" $D_{i}$, since it formally allows us to remove the $D_{i}$ from invariants. Note, however, that this equation is different in form from the usual divisor equation.

It is useful to see what the divisor equation tells us about the form of the potential function. For $Y$, it is well known that repeated application of the divisor equation implies that (up to unstable terms) we have

$$
F^{Y}=F^{Y}\left(y_{0}, 0,0, \ldots, 0, y_{r+1}, \ldots, y_{a}, q_{1} e^{y_{1}}, \ldots, q_{r} e^{y_{r}}\right) .
$$

In other words, the potential function depends on the variables in the combinations

$$
q_{1} e^{y_{1}}, \ldots, q_{r} e^{y_{r}}, y_{r+1}, \ldots, y_{n}
$$

We can apply this to only the exceptional classes, giving the form more useful to us here:

$$
F^{Y}=F^{Y}\left(y_{0}, \ldots, y_{s}, 0, \ldots, 0, y_{r+1}, \ldots, y_{a}, q_{1}, \ldots, q_{s}, q_{s+1} e^{y_{s+1}}, \ldots, q_{r} e^{y_{r}}\right)
$$

For the orbifold invariants, the analogous result is that the extended potential function depends on the variables only in the combinations

$$
u_{1} e^{x_{1}}, \ldots, u_{s} e^{x_{s}},\left(u_{s+1}+x_{s+1}\right), \ldots,\left(u_{r}+x_{r}\right), x_{r+1}, \ldots, x_{n} .
$$

More precisely, equation (1) implies the identity

$$
\widehat{F}^{\mathcal{X}}=F^{\mathcal{X}}\left(x_{0}, \ldots, x_{s},\left(x_{s+1}+u_{s+1}\right), \ldots,\left(x_{r}+u_{r}\right), x_{r+1}, \ldots, x_{n}, u_{1}, \ldots, u_{s}\right) .
$$

So, assuming Conjecture 1.2, we see that we get the equality $\widehat{F}^{\mathcal{X}}=F^{Y}$ for the extended potential function after the change of variables:

$$
\begin{aligned}
y_{i} & =\sum_{j} L_{i}^{j} x_{j} \\
q_{i} & = \begin{cases}c_{i} e^{L_{i}^{j} u_{j}} & \text { when } i>s \\
u_{i} & \text { when } i \leq s .\end{cases}
\end{aligned}
$$

Since this change of variables is invertible up to the discrete choices of branches of certain logarithms, it shows that one can essentially recover the Gromov-Witten theory of $Y$ from that of $\mathcal{X}$. Moreover, in this form it is especially clear that the existence of the standard divisor equation on $Y$ gives a very strong and mysterious 
prediction about the potential for $\mathcal{X}$ - it should depend on the new $u$ variables (or equivalently some of the original $x$ variables) only in terms of certain exponentials.

2.3. Orbifold Quantum Cohomology. Another application of the formalism of this section is to define a quantum product for an orbifold that is equivalent to the quantum product of its crepant resolution by a method completely parallel to the usual definition. We will discuss here only the small quantum cohomology. Of course, one can use the derivatives of the genus zero potential function to define a big quantum cohomology ring for orbifolds and everything we say can be applied there as well.

Assume $\mathcal{X}$ is a Gorenstein orbifold with projective coarse moduli scheme. We consider the three evaluation maps from $\bar{M}_{0,3}(\mathcal{X}, \widehat{\beta})$ to $I \mathcal{X}$, and given classes $\delta$ and $\gamma$ in $H^{*}(I \mathcal{X})$, we define

$$
\delta * \gamma=\sum_{\widehat{\beta}}\left(\langle\delta, \gamma, *\rangle_{\widehat{\beta}}^{\mathcal{X}}\right)^{\vee} u^{\widehat{\beta}}
$$

where $(\cdot)^{\vee}$ denotes dual with respect to the orbifold Poincaré pairing. The same proof of associativity holds for this product as for the one considered in [2].

We can express the quantum product in a basis, using the orbifold Poincaré pairing $g_{i j}$ on $H^{*}(I \mathcal{X})$ as

$$
\delta * \gamma=\sum\left\langle\delta, \gamma, \gamma_{i}\right\rangle_{\widehat{\beta}} g^{i j} \gamma_{j} u^{\widehat{\beta}}
$$

Hence, it is an immediate consequence of Conjecture 1.2 that the products agree in the sense that if we identify $H^{*}(Y)$ and $H^{*}(I \mathcal{X})$ using $L$, then the structure constants for the quantum product are related by the change of variables:

$$
q_{i} \mapsto \begin{cases}c_{i} e^{L_{i}^{j} u_{j}} & \text { when } i>s \\ u_{i} & \text { when } i \leq s .\end{cases}
$$

REMARK 2.4. As in subsection 1.4, this definition of the quantum product makes sense using only the hypothesis that $X$ is projective over an affine scheme. The argument reducing the equivalence of the quantum products of $\mathcal{X}$ and $Y$ to the equivalence of the potential functions uses the perfectness of the Poincaré pairing, which we do have in the torus equivariant setting provided that the fixed locus is compact.

REMARK 2.5. The definition of small quantum cohomology given in [15] or [2] can be recovered from this one by setting the new parameters equal to zero. It follows then, that one recovers that quantum cohomology ring of $\mathcal{X}$ from the quantum cohomology ring of $Y$ by simply setting some of the $q$ 's to roots of unity. The idea of setting quantum parameters on the resolution equal to roots of unity first appears in the mathematics literature in the work of Ruan [36] where he observes that in some examples, one needs to set $q=-1$ to recover the orbifold cohomology of $\mathcal{X}$.

\section{Examples}

To provide evidence for our conjecture we consider orbifolds of the form

$$
\mathcal{X}=[V / G]
$$

where $G \subset S L(V)$ is a finite subgroup. 
When the dimension of $V$ is 2 or 3 , there is a canonical crepant resolution given by the $G$-Hilbert scheme [7]:

$$
Y=\mathrm{G}-\mathrm{Hilb}(V) \text {. }
$$

The diagonal $\mathbb{C}^{\times}$action on $V$ commutes with $G$ and the induced action on $X$ lifts to $Y$. Thus the crepant resolution conjecture can be considered $\mathbb{C}^{\times}$equivariantly.

3.1. Polyhedral and Binary polyhedral groups. A finite subgroup $G$ of $S O(3)$ (respectively $S U(2)$ ) is called a polyhedral (respectively binary polyhedral) group. Such groups are classified by ADE Dynkin diagrams and they come with a natural representation $V$ of dimension 3 (respectively 2). For these groups, there is a canonical basis for $H_{\mathbb{C}^{*}}^{*}(\mathrm{G}-\mathrm{Hilb} V)$ indexed by $R \in \operatorname{Irr}(G)$, irreducible representations of $G[32,5]$. On the other hand, there is a canonical basis of $H_{\mathbb{C}^{*} \text {, orb }}^{*}([V / G])$ indexed by $(g) \in \operatorname{Conj}(G)$, conjugacy classes of $G$. Denote the corresponding cohomology variables by

$$
\left\{y_{R}\right\}_{R \in \operatorname{Irr}(G)} \text { and }\left\{x_{(g)}\right\}_{(g) \in \operatorname{Conj}(G)}
$$

respectively. Let $y_{0}$ and $x_{0}$ be the variables corresponding to the trivial representation and the trivial conjugacy class respectively.

The equivariant quantum cohomology of G-Hilb $(V)$ has been completely described in terms of the root theory of the corresponding ADE root system by Bryan-Gholampour $[\mathbf{9}, \mathbf{1 0}]$. They conjecture that the change of variables for the crepant resolution is a certain modification of the character table:

CONJECTURE 3.1. The change of variables for the crepant resolution conjecture in the case of

$$
\mathrm{G}-\mathrm{Hilb}(V) \rightarrow V / G
$$

where $G$ is a polyhedral or binary polyhedral group is given by

$$
\begin{aligned}
y_{0} & =x_{0}, \\
y_{R} & =\frac{1}{|G|} \sum_{g \in G} \sqrt{\chi_{V}(g)-\operatorname{dim} V} \chi_{R}(g) x_{(g)}, \\
q_{R} & =\exp \left(\frac{2 \pi i \operatorname{dim} R}{|G|}\right)
\end{aligned}
$$

where $R$ runs over the non-trivial irreducible representations of $G$.

The term under the radical is always a negative real number and so the square root can chosen to be a positive multiple of $i$. Note that as a consequence of $V$ being the natural representation of a polyhedral or binary polyhedral group, the orbifold $\mathcal{X}=[V / G]$ satisfies the hard Lefschetz condition. Moreover, all non-trivial conjugacy classes have age one, and so the above linear transformation preserves the grading. In fact, up to summands of the trivial representation, these are the only faithful group representations that have the property that all non-trivial elements have age one.

Using the root theoretic formula for the Gromov-Witten potential of G-Hilb $(V)$ given in $[\mathbf{9}, \mathbf{1 0}]$ and applying the crepant resolution to the above change of variables, one arrives at a prediction for the orbifold Gromov-Witten potential $F^{\mathcal{X}}$. This prediction has not been verified in general, but it does pass some strong tests of its validity. Namely, it can be shown to exhibit various vanishing properties and to have the correct classical terms. 
The complete determination of $F^{\mathcal{X}}$, and hence the verification of the crepant resolution conjecture, has been done for $G$ equal to

$$
\begin{aligned}
& \mathbb{Z}_{2} \subset S U(2) \text { in the next subsection, } \\
& \mathbb{Z}_{3} \subset S U(2) \text { in }[\mathbf{1 1}], \\
& \mathbb{Z}_{4} \subset S U(2) \text { in }[\mathbf{1 2}], \\
& \mathbb{Z}_{2} \times \mathbb{Z}_{2} \subset S O(3) \text { in }[\mathbf{8}], \text { and } \\
& A_{4} \subset S O(3) \text { in }[\mathbf{8}] .
\end{aligned}
$$

Recently, the case of $\mathbb{Z}_{n} \subset S U(2)$ was done for all $n$ by Coates, Corti, Iritani, and Tseng [18].

3.2. The case of the rational double point. We consider the case where $V=\mathbb{C}^{2}$ and $G=\{ \pm 1\} \subset S U(2)$ so that

$$
\mathcal{X}=\left[\mathbb{C}^{2} /\{ \pm 1\}\right], \quad Y=T^{*} \mathbb{P}^{1} .
$$

This is the simplest nontrivial example and it already provides a very interesting case study. Here we will establish the equivariant version of the conjecture.

Let $T=\mathbb{C}^{\times} \times \mathbb{C}^{\times}$so that

$$
H_{T}^{*}(\mathrm{pt}) \cong \mathbb{Q}\left[t_{1}, t_{2}\right]
$$

The natural $T$ action on $\mathbb{C}^{2}$ induces a $T$ action on $Y$, the minimal resolution of the quotient $X=\mathbb{C}^{2} /\{ \pm 1\} . Y$ is isomorphic to $T^{*} \mathbb{P}^{1}$, the total space of the cotangent bundle of $\mathbb{P}^{1}$. There are two fixed points of the $T$ action on $Y$ having weights

$$
\left(2 t_{1}, t_{2}-t_{1}\right) \text { and }\left(2 t_{2}, t_{1}-t_{2}\right) \text {. }
$$

First we will compute the genus zero potential function for $Y$. We take our generator for $H_{2}(Y)$ to be the class of the zero section, $[E]$. We let $\gamma \in H_{T}^{2}(Y)$ be the dual of $[E]$, so that $\int_{E} \gamma=1$. It is given by the first Chern class of an equivariant line bundle with weights $-t_{1}$ and $-t_{2}$ at the fixed points.

The degree zero invariants are simply given by triple intersections in equivariant cohomology

$$
\langle a, b, c\rangle_{0}=\int_{Y} a \cup b \cup c
$$

which are computed by localization. The results are:

$$
\langle 1,1,1\rangle_{0}=\frac{1}{2 t_{1} t_{2}}, \quad\langle\gamma, 1,1\rangle_{0}=0, \quad\langle\gamma, \gamma, 1\rangle_{0}=-\frac{1}{2}, \quad\langle\gamma, \gamma, \gamma\rangle_{0}=\frac{1}{2}\left(t_{1}+t_{2}\right)
$$

To compute the invariants in positive degrees, we first observe that the image of any nonconstant morphism from a curve to $Y$ must lie in $E$. Thus we have a natural isomorphism

$$
\bar{M}_{0, n}(Y, d[E]) \cong \bar{M}_{0, n}\left(\mathbb{P}^{1}, d\right)
$$

however, the virtual fundamental classes on the two sides differ. Under the above identification, it is well known that

$$
\left[\bar{M}_{0, n}(Y, d[E])\right]^{\text {vir }}=e\left(R^{1} \pi_{*} f^{*} N_{E / Y}\right)
$$

where $\pi: \mathcal{C} \rightarrow \bar{M}_{0, n}\left(\mathbb{P}^{1}, d\right)$ and $f: \mathcal{C} \rightarrow Y$ are the universal curve and the universal map respectively. 
Since $E$ is a -2 curve, we have an isomorphism of $N_{E / Y} \cong \mathcal{O}(-2)$. Consider the standard Euler sequence on $\mathbb{P}^{1}$,

$$
0 \rightarrow \mathcal{O}(-2) \rightarrow \mathcal{O}(-1) \oplus \mathcal{O}(-1) \rightarrow \mathcal{O} \rightarrow 0
$$

Pulling this sequence back to $\mathcal{C}$ and taking the associated long exact sequence of derived pushforwards gives us

$$
0 \rightarrow \mathcal{O} \rightarrow R^{1} \pi_{*} f^{*}(\mathcal{O}(-2)) \rightarrow R^{1} \pi_{*} f^{*}(\mathcal{O}(-1) \oplus \mathcal{O}(-1)) \rightarrow 0 .
$$

An analysis of the weights shows that the action of $T$ on the left hand term in this sequence is given by $t_{1}+t_{2}$. We conclude that

$$
e\left(R^{1} \pi_{*} f^{*} N_{E / Y}\right)=\left(t_{1}+t_{2}\right) e\left(R^{1} \pi_{*}(\mathcal{O}(-1)+\mathcal{O}(-1))\right)
$$

The integral is then evaluated using the famous Aspinwall-Morrison formula:

$$
\langle\rangle_{d}=\left(t_{1}+t_{2}\right) \int_{\left[\bar{M}_{0,0}\left(\mathbb{P}^{1}, d\right)\right]} e\left(R^{1} \pi_{*} f^{*}(\mathcal{O}(-1) \oplus \mathcal{O}(-1))\right)=\frac{t_{1}+t_{2}}{d^{3}} .
$$

Let $y_{0}$ and $y_{1}$ denote the variables corresponding to 1 and $\gamma$. Combining the above formulas with the divisor equation and the point axiom, we have shown the following.

Proposition 3.2. The genus zero Gromov-Witten potential function of $Y$ is given by:

$$
F^{Y}=\frac{1}{12 t_{1} t_{2}} y_{0}^{3}-\frac{1}{4} y_{0} y_{1}^{2}+\frac{t_{1}+t_{2}}{12} y_{1}^{3}+\left(t_{1}+t_{2}\right) \sum_{d>0} \frac{1}{d^{3}} q^{d} e^{d y_{1}}
$$

We now consider the invariants for the orbifold $\mathcal{X}$. Let 1 and $D$ be the natural generators for $H_{o r b}^{0}(\mathcal{X})$ and $H_{o r b}^{2}(\mathcal{X})$ and let $x_{0}$ and $x_{1}$ be the corresponding variables.

Since the coarse moduli space for $\mathcal{X}$ is affine, every stable map is constant. If the source curve has any twisted points, the image of the map is forced to be the unique point of $\mathcal{X}$ with nontrivial stabilizer. Thus we see that with the exception of

$$
\langle 1,1,1\rangle=\frac{1}{2 t_{1} t_{2}}
$$

every invariant naturally arises as an integral over $\bar{M}_{0, n}\left(B \mathbb{Z}_{2}\right)$. By the point axiom and monodromy considerations, the only other invariant involving 1 is

$$
\langle 1, D, D\rangle=\frac{1}{2} \text {. }
$$

Since the only remaining non-vanishing invariants are then $\left\langle D^{n}\right\rangle$ we actually need only consider the connected component of $\bar{M}_{0, n}\left(B \mathbb{Z}_{2}\right)$ where all the evaluation maps go to the twisted sector. Setting $n=2 g+2$, we denote this space as $\bar{H}_{g}^{\text {ord }}$. Concretely, it is the usual compactified moduli space of hyperelliptic curves (with ordered branch points). The virtual class on $\bar{H}_{g}^{\text {ord }}$ is given by

$$
e\left(R^{1} \pi_{*} f^{*}(L \oplus L)\right)
$$

where $f$ and $\pi$ are the universal map and universal curve for $\bar{M}_{0, n}\left(B \mathbb{Z}_{2}\right)$ and

$$
L \oplus L \rightarrow B \mathbb{Z}_{2}
$$

is two copies of the non-trivial line bundle over $B \mathbb{Z}_{2}$ with the torus acting with weight $t_{1}$ on the first factor and with weight $t_{2}$ on the second factor. The bundle 
$R^{1} \pi_{*} f^{*} L$ is in fact isomorphic to $\mathbb{E}^{\vee}$, the dual of the Hodge bundle pulled back by the map $\bar{H}_{g}^{\text {ord }} \rightarrow \bar{M}_{g}$. We conclude that that for $n=2 g+2>0$, we can write

$$
\begin{aligned}
\left\langle D^{n}\right\rangle & =\int_{\bar{H}_{g}^{\text {ord }}} e\left(\mathbb{E}^{\vee} \oplus \mathbb{E}^{\vee}\right) \\
& =-\left(t_{1}+t_{2}\right) \int_{\bar{H}_{g}^{\text {ord }}} \lambda_{g} \lambda_{g-1} .
\end{aligned}
$$

The generating function for these integrals was computed in [22, Corollary 2]. Applying that computation, we obtain:

Proposition 3.3. The potential function of $\mathcal{X}=\left[\mathbb{C}^{2} /\{ \pm 1\}\right]$ is given by

$$
F^{\mathcal{X}}\left(x_{0}, x_{1}\right)=\frac{1}{12 t_{1} t_{2}} x_{0}^{3}+\frac{1}{4} x_{0} x_{1}^{2}-\left(t_{1}+t_{2}\right) x_{1}^{2} H\left(x_{1}\right)
$$

where (following the notation of [22]) $H\left(x_{1}\right)$ satisfies

$$
\left(x_{1}^{2} H\left(x_{1}\right)\right)^{\prime \prime \prime}=\frac{1}{2} \tan \left(\frac{x_{1}}{2}\right) .
$$

COROLLARY 3.4. The crepant resolution conjecture holds for the pair $(Y, \mathcal{X})$. That is, the potential functions $F^{Y}\left(y_{0}, y_{1}, q\right)$ and $F^{X}\left(x_{0}, x_{1}\right)$ agree, up to unstable terms, under the change of variables (c.f. Conjecture 3.1)

$$
y_{0}=x_{0}, \quad y_{1}=i x_{1}, \quad q=-1 .
$$

Proof: Clearly the terms of $F^{Y}$ and $F^{\mathcal{X}}$ which have $y_{0}$ and $x_{0}$ match up. And since we are only interested in stable terms, it suffices to check that

$$
\left(\frac{d}{d x_{1}}\right)^{3} F^{Y}\left(x_{0}, i x_{1},-1\right)=\left(\frac{d}{d x_{1}}\right)^{3} F^{\mathcal{X}}\left(x_{0}, x_{1}\right) .
$$

The right hand side is given by

$$
-\left(t_{1}+t_{2}\right) \frac{1}{2} \tan \left(\frac{x_{1}}{2}\right)
$$

whereas the left hand side is

$$
\begin{aligned}
& \left(t_{1}+t_{2}\right)\left[\frac{i^{3}}{2}+\sum_{d=1}^{\infty} i^{3}\left(-e^{i x_{1}}\right)^{d}\right] \\
= & \left(t_{1}+t_{2}\right) \frac{1}{2 i}\left[\frac{1-e^{i x_{1}}}{1+e^{i x_{1}}}\right] \\
= & \left(t_{1}+t_{2}\right) \frac{1}{2} \tan \left(\frac{-x_{1}}{2}\right) .
\end{aligned}
$$

3.3. The case of the Hilbert scheme. We consider the case where

$$
\mathcal{X}=\operatorname{Sym}^{n}\left(\mathbb{C}^{2}\right) \text { and } Y=\operatorname{Hilb}^{n}\left(\mathbb{C}^{2}\right) .
$$

This is one of the best known and most studied examples of a crepant resolution of singularities of a Gorenstein orbifold.

We will show in this section that by matching the Nakajima basis for the cohomology of the Hilbert scheme with the natural basis for the orbifold cohomology of the symmetric product we verify Conjecture 1.2 in this case. 
Because the Hilbert scheme is holomorphically symplectic, there are no interesting Gromov-Witten invariants unless one works equivariantly. An analogous fact is true on the orbifold side. Thus, to verify the conjecture for the nonequivariant theory it suffices to compare the ring structure on the ordinary cohomology of the Hilbert scheme with the orbifold cohomology of the symmetric product. This is done in $[39,29]$ (see also $[23,37]$ ).

The nontrivial, fully equivariant genus 0 Gromov-Witten theory of $Y$ is determined in [34]. We will determine the genus 0 equivariant Gromov-Witten theory of $\mathcal{X}$ and verify that it matches their result after the appropriate change of variables.

First, let us describe the inertia stack $I \mathcal{X}$. We use the standard correspondence between conjugacy classes of $S_{n}$ and partitions of $n$. Given such a partition $\mu$, the corresponding component of the inertia stack $I_{\mu}$ can be described by choosing a representative permutation $\sigma$ and taking the stack quotient $\left[\mathbb{C}_{\sigma}^{2 n} / C(\sigma)\right]$ where $\mathbb{C}_{\sigma}^{2 n}$ denotes the invariant part of $\mathbb{C}^{2 n}$ under the action of $\sigma$ and $C(\sigma)$ denotes the centralizer of $\sigma$ in $S_{n}$. The dimension of $\mathbb{C}_{\sigma}^{2 n}$ is $2 l(\mu)$ and the age of $\mu$ is $n-l(\mu)$. The quotient of a vector space by a finite group has no higher cohomology groups, so we conclude that a basis for the orbifold cohomology of $\mathcal{X}$ as a $\mathbb{Q}\left[t_{1}, t_{2}\right]$-module is given by

$$
\left[I_{\mu}\right] \subset H_{T, \text { orb }}^{2 n-2 l(\mu)}(\mathcal{X})
$$

Since each element of $S_{n}$ is conjugate to its inverse, the equivariant Poincaré pairing on $H_{T, \text { orb }}^{*}(\mathcal{X})$ is diagonal in this basis. It is easily computed by localization, since the fixed points of the $T$ action on $I \mathcal{X}$ are isolated - there is a single fixed point in each irreducible component $I_{\mu}$. This point has automorphism group equal to the centralizer of a representative element, which has order

$$
\mathfrak{z}(\mu)=|\operatorname{Aut}(\mu)| \prod \mu_{i}
$$

It follows that the pairing is given by

$$
\left(\left[I_{\mu}\right],\left[I_{\mu}\right]\right)=\frac{1}{\mathfrak{z}(\mu)}\left(t_{1} t_{2}\right)^{-l(\mu)} .
$$

It is straightforward to check that the orbifold product here is a slight modification of the usual multiplication on $Z \mathbb{Q}\left[S_{n}\right]$, the center of the group ring of $S_{n}$ obtained by inserting factors of $t_{1} t_{2}$ to make that product respect the grading by age. In particular, the limit $t_{1}=t_{2}=1$ gives the standard product on $Z \mathbb{Q}\left[S_{n}\right]$.

On the Hilbert scheme, there is an analogous description of the equivariant cohomology, given by the Nakajima basis. Given a partition $\mu$, the corresponding class

$$
N_{\mu} \in H_{T}^{2 n-2 l(\mu)}\left(\operatorname{Hilb}^{n}\left(\mathbb{C}^{2}\right)\right)
$$

is given by $\frac{1}{\prod \mu_{i}}\left[C_{\mu}\right]$ where $C_{\mu}$ is the subvariety of $\operatorname{Hilb}^{n}\left(\mathbb{C}^{2}\right)$ whose general point parameterizes a length $n$ subscheme composed of $l(\mu)$ irreducible components of lengths $\mu_{i}$. The $T$-equivariant Poincaré pairing in the Nakajima basis is also diagonal with

$$
\left(N_{\mu}, N_{\mu}\right)=\frac{(-1)^{n-l(\mu)}}{\mathfrak{z}(\mu)}\left(t_{1} t_{2}\right)^{-l(\mu)}
$$

This gives us an obvious candidate for the map $L$ identifying the orbifold cohomology of $\operatorname{Sym}^{n}\left(\mathbb{C}^{2}\right)$ with the cohomology of Hilb ${ }^{n}\left(\mathbb{C}^{2}\right)$. Namely, we define $L$ by

$$
L\left(\left[I_{\mu}\right]\right)=i^{n-l(\mu)} N_{\mu}
$$


Note also, that since there exists a unique partition of length $n-1$, the partition corresponding to a 2-cycle, which we will denote (2), there is only one divisor class, and so a single constant $c$ to choose to finish determining the change of variables. The correct choice of $c$ turns out to be -1 . Thus the predicted change of variables for the quantum parameters is

$$
q=-e^{i u}
$$

To establish the full equality of the genus zero Gromov-Witten potentials, it will be extremely convenient to use the formalism introduced for the small quantum product as a bookkeeping device. Let $c_{\mu}^{\nu}$ be the structure constants for quantum multiplication by $\left[I_{(2)}\right]$ :

$$
\left[I_{(2)}\right] *\left[I_{\mu}\right]=\sum_{\nu} c_{\mu}^{\nu}\left[I_{\nu}\right]
$$

Here the $c_{\mu}^{\nu}$ are elements of $\mathbb{Q}\left[t_{1}, t_{2}\right][[u]]$ where $u$ is the quantum parameter associated to the twisted sector as defined in Section 2. If we let $c_{\mu}^{\nu}(d)$ denote the coefficient of $u^{d}$ in $c_{\mu}^{\nu}$, then we have the formula

$$
c_{\mu}^{\nu}(d)=\mathfrak{z}(\nu)\left(t_{1} t_{2}\right)^{l(\nu)}\left\langle\left[I_{\mu}\right],\left[I_{\nu}\right],\left[I_{(2)}\right]\right\rangle_{d} .
$$

Note that the above Gromov-Witten invariant is an element of $\mathbb{Q}\left(t_{1}, t_{2}\right)$, whereas $c_{\mu}^{\nu}(d)$ is a polynomial. This fact will be essential for the degree arguments that follow. While the polynomality is an immediate consequence of the existence of the equivariant quantum product referred to in Section 1.4, the reader can also check that it follows directly from the explicit localization formula we will give in the next section.

By degree considerations, we see that $c_{\mu}^{\nu}$ vanishes if $l(\mu) \geq l(\nu)+1$. Since Equation 3 gives a symmetry, we also have the inequality $l(\nu) \geq l(\mu)+1$. We will see below that if $|l(\nu)-l(\mu)|=1$ then the only contribution to $c_{\mu}^{\nu}$ is in degree zero where we just see the classical term corresponding to multiplication in the group ring of $S_{n}$. Aside from these classical terms, the matrix $c_{\mu}^{\nu}$ is diagonal.

LEMMA 3.5. If $l(\mu)=l(\nu)$, but $\mu \neq \nu$, then $c_{\mu}^{\nu}=0$.

Proof: Let $\bar{M}_{0,(\lambda, \mu, \nu)}(\mathcal{X}, d)$ denote the component(s) of $\bar{M}_{0,3}(\mathcal{X}, d)$ given by $e_{1}^{-1}\left(I_{\lambda}\right) \cap e_{2}^{-1}\left(I_{\mu}\right) \cap e_{3}^{-1}\left(I_{\nu}\right)$.

By definition, we have

$$
c_{\mu}^{\nu}(d)\left[I_{\nu}\right]=e_{3 *}\left(\left[\bar{M}_{0,((2), \mu, \nu)}(\mathcal{X}, d)\right]^{\text {vir }}\right)^{\vee} .
$$

By degree consideration, this must be a codimension 1 class in $I_{\nu}$. However it is easy to see that the codimension of $e_{3}\left(\bar{M}_{0,((2), \mu, \nu)}(\mathcal{X}, d)\right)$ is at least 2 , since the intersection of the images of $I_{\mu}$ and $I_{\nu}$ in $\mathcal{X}$ has codimension at least 2 in each. The lemma follows immediately.

To finish the determination of the structure of the quantum cohomology ring, we use a localization calculation. Because $\operatorname{Sym}^{n}\left(\mathbb{C}^{2}\right)$ is affine, every twisted stable map is constant at the level of coarse moduli schemes. It follows that we have a canonical identification of the $T$ fixed locus of the space of maps to $\mathcal{X}$ with the space of maps to $B S_{n}$ (the fixed locus of the action of $T$ on $\mathcal{X}$ ). The normal bundle to this fixed locus decomposes naturally as a sum of two rank $n$ vector bundles. These two bundles come with $T$ weights $t_{1}$ and $t_{2}$, but are otherwise identical, each corresponding to the standard $n$-dimensional representation of $S_{n}$ under the usual correspondence between sheaves on $B G$ and representations of $G$. We will use $V$ to 
denote this bundle. There is another way to think of $V$ which is convenient for us here. Consider the morphism $i: B S_{n-1} \rightarrow B S_{n}$ induced by the standard inclusion of $S_{n-1} \hookrightarrow S_{n}$. Then $V$ is simply the pushforward of the structure sheaf.

Since the coarse moduli scheme of $B S_{n}$ is a point, the moduli space of twisted maps $\bar{M}_{0, r}\left(B S_{n}\right)$ is smooth of dimension $r-3$. By the results of [26] we can identify the equivariant virtual fundamental class of $\bar{M}_{0, r}\left(\operatorname{Sym}^{n}\left(\mathbb{C}^{2}\right)\right)$ with the pushforward from this fixed locus of the class

$$
e\left(-R^{\bullet} \pi_{*} f^{*}(V \oplus V)\right)
$$

where the torus acts on the two factors of $V$ are with weights $t_{1}$ and $t_{2}$, and where $f$ and $\pi$ are the universal maps in the universal diagram

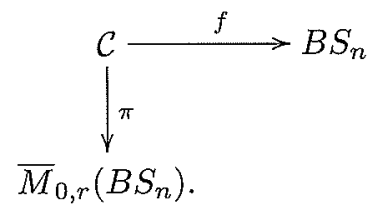

To give a description of the virtual class in more familiar terms, we extend the above diagram to

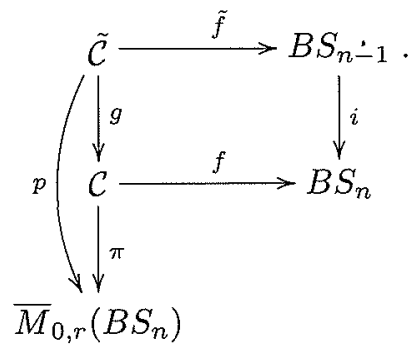

The curve $\tilde{\mathcal{C}}$ here is the degree $n$ covering of $\mathcal{C}$ corresponding to the map to $B S_{n}$ via the usual correspondence between principal $S_{n}$ bundles and degree $n$ étale covers.

We know that $V=i_{*} \mathcal{O}_{B S_{n-1}}$. Since $i$ is a finite morphism, we have $f^{*}\left(i_{*} \mathcal{O}\right)=$ $g_{*} \tilde{f}^{*} \mathcal{O}_{B S_{n-1}}=g_{*} \mathcal{O}_{\tilde{C}}$. Since $g_{*}$ is exact, we conclude that the virtual class can be rewritten as the pushforward from the fixed locus of

$$
e\left(-R^{\bullet} p_{*}\left(\mathcal{O}_{\tilde{\mathcal{C}}} \oplus \mathcal{O}_{\tilde{\mathcal{C}}}\right)\right)
$$

In other words, if we think of the space of maps to $B S_{n}$ as parameterizing the family of $n$-sheeted covers of $\mathbb{P}^{1}$ given by $\tilde{\mathcal{C}}$, then the invariants we want to compute are expressed in terms of the Chern classes of the Hodge bundle. Thus if we let

$$
\mathbb{E}^{\vee}=R^{1} p_{*} \mathcal{O}_{\tilde{\mathcal{C}}}
$$

and we let $s$ denote the locally constant function on $\bar{M}_{0, r}\left(B S_{n}\right)$ recording the number of connected components of the fibers of $\tilde{\mathcal{C}}$, we obtain the following formula.

LEMMA 3.6. The $r$ point, degree zero invariants of $\mathcal{X}$ are given by

$$
\left\langle\mu_{1}, \ldots, \mu_{r}\right\rangle_{0}=\int_{\left[\bar{M}_{0,\left(\mu_{1}, \ldots, \mu_{r}\right)}\left(B S_{n}\right)\right]^{\mathrm{vir}}}\left(t_{1} t_{2}\right)^{-s} c_{t o p}\left(\mathbb{E}_{t_{1}}^{\vee} \oplus \mathbb{E}_{t_{2}}^{\vee}\right)
$$


where $\bar{M}_{0,\left(\mu_{1} \cdots \mu_{r}\right)}\left(B S_{n}\right)$ is the component(s) of $\bar{M}_{0, r}\left(B S_{n}\right)$ where $e_{i}$ maps to the component of $I B S_{n}$ corresponding to $\mu_{i}$. We also must interpret the above integral as a sum over connected components of the moduli space. The rank of $\mathbb{E}$ and the integer $s$ can vary from component to component.

Since the orbifold $\mathcal{X}$ has divisor classes only in the twisted sector, the higher degree invariants are determined by the degree zero invariants by the divisor equation. Thus the above lemma determines all the invariants.

LEMMA 3.7. If $d>0$, then $c_{\mu}^{\nu}(d)$ is divisible by $\left(t_{1}+t_{2}\right)$.

This is a consequence of Mumford's relation that $c\left(\mathbb{E} \oplus \mathbb{E}^{\vee}\right)=1$. If we set $t_{2}=-t_{1}$ and use the fact that $c_{\text {top }}\left(\mathbb{E}_{-t_{1}}^{\vee}\right)= \pm c_{\text {top }}\left(\mathbb{E}_{t_{1}}\right)$, we find that the integrand in Lemma 3.6 is simply a power of $t_{1}$. The hypothesis $d>0$ implies that the moduli space is positive dimensional, so the result follows.

We remark that on the Hilbert scheme side, this divisibility is related to the existence of a holomorphic symplectic structure on $\mathrm{Hilb}^{n}\left(\mathbb{C}^{2}\right)$.

Corollary 3.8. If $d>0$ and $\mu \neq \nu$, we have $c_{\mu}^{\nu}=0$.

Given Lemma 3.5 and the discussion just before it, we see that the only interesting case here is if $l(\mu)=l(\nu)+1$. However, in this case, we know that the degree of $c_{\mu}^{\nu}$ is zero, so the divisibility constraint forces this invariant to vanish.

This reduces our task to the calculation of the invariants $c_{\mu}^{\mu}$. We can further reduce to the case where the partition $\mu$ has just one part, by using the following lemma.

LEMMA 3.9.

$$
c_{\mu}^{\mu}=\frac{1}{\mathfrak{z}(\mu)} \sum_{i=1}^{l(\mu)} \mu_{i} c_{\left(\mu_{i}\right)}^{\left(\mu_{i}\right)} .
$$

The right hand side of this formula is easily seen to be the contribution from those components of $\bar{M}_{0,((2), \mu, \mu)}(\mathcal{X}, d)$ where the corresponding branched cover $C$ consists of $l(\mu)$ connected components, all but one of which is a smooth genus zero curve branched only at 0 and $\infty$. To prove the lemma, we need to show that the other components make no contribution. We will do this by means of the formula of Lemma 3.6, so we will always be considering the space of maps to $B S_{n}$ rather than to $\mathcal{X}$, and we will denote the two distinguished points of the source curve corresponding to $\mu$ as 0 and $\infty$. These are the only points over which the associated branched cover of $\mathbb{P}^{1}$ has non-simple branching.

Step 1: Suppose we have a component where the associated cover is connected. Then, by the Riemann-Hurwitz formula, it will have genus $g=\frac{d+3}{2}-l(\mu)$. In order for the integral in Lemma 3.6 not to vanish, it is obviously necessary that $2 g \geq d$ since $d$ is the dimension of the moduli space. This inequality is satisfied only if $l(\mu)=1$ (in which case the Lemma is vacuously true). Otherwise, we conclude that a component of the moduli space can contribute to this invariant only if it parameterizes disconnected covers.

Step 2: Suppose we consider a component $\bar{M}^{\prime}$ of the moduli space where the corresponding branched cover is disconnected. We get a natural map $\Psi: \bar{M}^{\prime} \rightarrow$ $\left(\prod_{a} \bar{M}_{a}\right) /$ Aut where the $\bar{M}_{a}$ are some moduli spaces of lower degree branched covers with certain branching conditions and the group Aut is acting by permuting factors with identical parameters. We do not need a very careful description 
here, since we will use just two crude facts. First, if two different factors of the target space parameterize covers with branching away from zero and $\infty$, then $\Psi$ has positive dimensional fibers, since we can independently act by $\mathbb{C}^{\times}$on different components. Since $\mathbb{E}$ is pulled back under $\Psi$ this immediately kills contributions from any such component of $\bar{M}$.

If a branched cover has all the simple branch points on a single connected component, then the other components are necessarily genus zero curves ramified only at zero and infinity. Now Step 1 will apply to the remaining interesting component, showing that this component of moduli space makes no contribution to the integral unless we are in the maximally disconnected case.

We remark that the argument in this lemma extends to give an alternate proof of Lemma 3.5 .

We see then, that we will have completely determined the quantum multiplication by $\left[I_{(2)}\right]$ once we calculate the invariants $c_{(n)}^{(n)}$ for all $n$. Here we can give an explicit formula.

LEMMA 3.10. We have the following:

$$
\sum_{d}\left\langle\left[I_{(n)}\right],\left[I_{(n)}\right],\left[I_{(2)}\right]\right\rangle_{d} u^{d}=\frac{1}{2} \frac{t_{1}+t_{2}}{t_{1} t_{2}}\left(n \cot \left(\frac{n u}{2}\right)-\cot \left(\frac{u}{2}\right)\right) .
$$

Proof: This follows from the same argument as [13, Theorem 6.5]. The restriction of $\tilde{\mathcal{C}}$ to $e_{1}^{-1}\left(I_{(n)}\right) \subset \bar{M}_{0,3}(\mathcal{X}, d)$ is necessarily a family of connected curves of genus $g$ where $d=2 g-1$. Applying Lemma 3.6 and the divisor equation, we get the formula

$$
\left\langle\left[I_{(n)}\right],\left[I_{(n)}\right],\left[I_{(2)}\right]\right\rangle_{d}=-\frac{t_{1}+t_{2}}{t_{1} t_{2}} \frac{1}{(2 g-1) !} \int_{\left[\bar{M}_{0,((n)(n)(2) \cdots(2))}\left(B S_{n}\right)\right]} \lambda_{g} \lambda_{g-1} .
$$

The map to $\bar{M}_{g, 2}$ induced by the family $\tilde{\mathcal{C}}$ is generically finite of degree $(2 g)$ ! onto its image, which is the set of curves admitting a degree $n$ map to $\mathbb{P}^{1}$ totally ramified at the two marked points. The image of this map is called $\bar{H}_{n} \subset \bar{M}_{g, 2}$ in [13] and the pairing of $\left[\bar{H}_{n}\right]$ against $\lambda_{g} \lambda_{g-1}$ is explicitly evaluated in [13] to yield the series above.

Having completely determined the $c_{\mu}^{\nu}$ we can deduce our main result.

THEOREM 3.11. After making the change of variables given by equation (2) and relating the quantum parameters by

$$
q=-e^{i u}
$$

the genus zero Gromov-Witten potential of $\mathrm{Hilb}^{n}\left(\mathbb{C}^{2}\right)$ is equal to the (extended) genus zero Gromov-Witten potential of $\operatorname{Sym}^{n}\left(\mathbb{C}^{2}\right)$. Hence the crepant resolution conjecture holds in this case.

By direct inspection, the matrix of multiplication by $\left[I_{(2)}\right]$ in $Q H^{*}\left(\operatorname{Sym}^{n}\left(\mathbb{C}^{2}\right)\right)$ matches with the matrix of multiplication by $i\left[N_{(2)}\right]$ in $Q H^{*}\left(\operatorname{Hilb}^{n}\left(\mathbb{C}^{2}\right)\right)$ calculated in [34] (equations (6) and (8), see also [13] equations (19) and (29)) under the change of variables $q=-e^{i u}$. As is observed there, the fact that this matrix has distinct eigenvalues implies that after extending the scalars to $\mathbb{Q}\left(t_{1}, t_{2}\right)$ the quantum cohomology is generated by the divisor class $\left[I_{(2)}\right]$. Thus the entire ring structure is encoded in this multiplication matrix. 
Finally, since the small quantum cohomology is generated by divisors, a variant of the reconstruction theorem of Kontsevich-Manin shows that one can use the WDVV equation to reduce arbitrary genus zero Gromov-Witten invariants to invariants with only two insertions (c.f. [35]). As these are already encoded in the small quantum product, the proof of the theorem is complete.

3.4. Equivalence with other theories. There are two other theories which are equivalent to the quantum cohomologies of $\operatorname{Sym}^{n}\left(\mathbb{C}^{2}\right)$ and $\mathrm{Hilb}^{n}\left(\mathbb{C}^{2}\right)$. By computing the equivariant Gromov-Witten partition function (in all genus) for the degree $n$ invariants of $\mathbb{P}^{1} \times \mathbb{C}^{2}$ relative to $\{0,1, \infty\} \times \mathbb{C}^{2}$, one obtains the structure constants of an associative Frobenius algebra [13]. Similarly, one obtains a Frobenius algebra from the partition function for the degree $n$ equivariant Donaldson-Thomas invariants of $\mathbb{P}^{1} \times \mathbb{C}^{2}$ relative to $\{0,1, \infty\} \times \mathbb{C}^{2}[\mathbf{3 3}]$. Theorem 3.11 completes the following tetrahedron of equivalences.

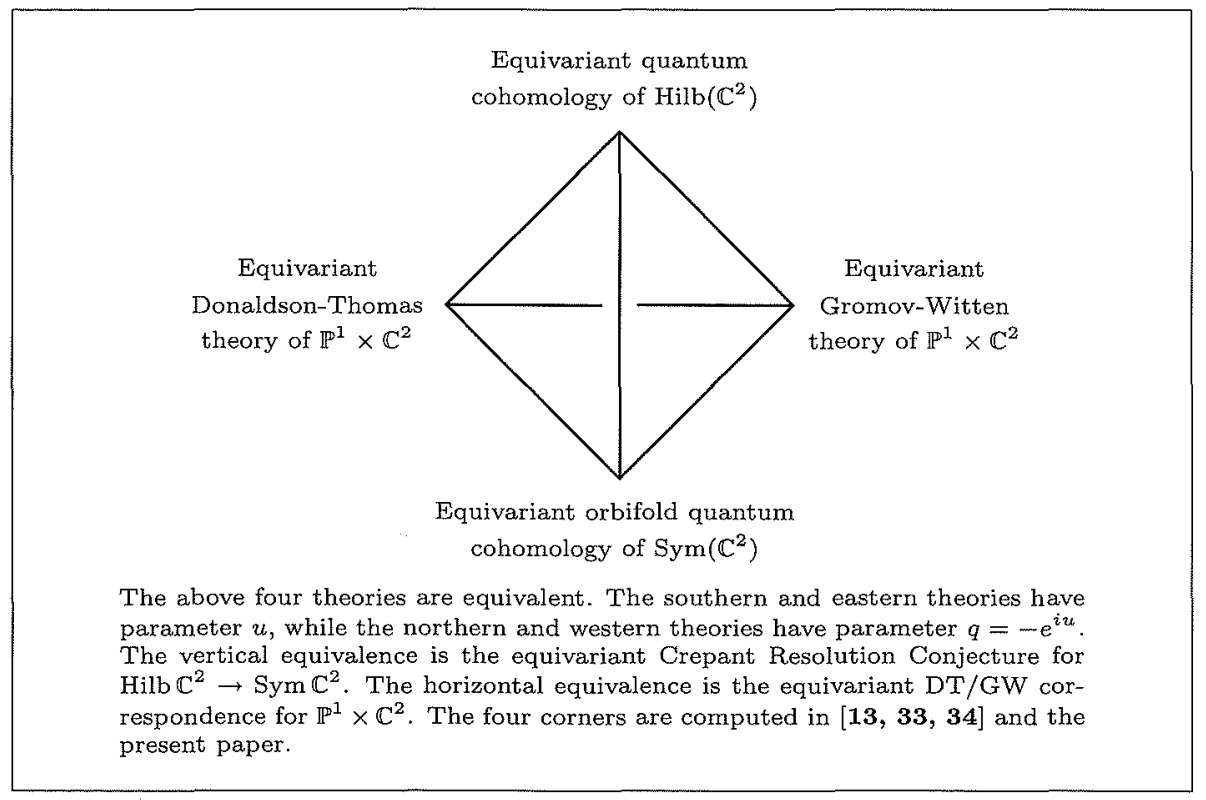

REMARK 3.12. A mysterious conjectural symmetry of Donaldson-Thomas theory is the invariance of the reduced Donaldson-Thomas partition function under $q \mapsto q^{-1}[30$, Conjecture 2]. In the examples of the above diagram, this symmetry arises from the invariance of the potential functions under $i u \mapsto-i u$. This is an example of the symmetry arising from the action of the Galois group on the change of variables as in remark 1.7.

3.5. Acknowledgments. We warmly acknowledge helpful discussions with Mina Aganagic, Renzo Cavalieri, Tom Coates, Amin Gholampour, Yunfeng Jiang, Rahul Pandharipande, Michael Thaddeus, and Hsian-Hua Tseng. We acknowledge support from NSERC, NSF, the Sloan Foundation, and IHES. 


\section{References}

[1] Dan Abramovich, Tom Graber, and Angelo Vistoli. Gromov-Witten theory of DeligneMumford stacks. arXiv:math.AG/0603151.

[2] Dan Abramovich, Tom Graber, and Angelo Vistoli. Algebraic orbifold quantum products. In Orbifolds in mathematics and physics (Madison, WI, 2001), volume 310 of Contemp. Math., pages 1-24. Amer. Math. Soc., Providence, RI, 2002.

[3] Dan Abramovich and Angelo Vistoli. Compactifying the space of stable maps. J. Amer. Math. Soc., 15(1):27-75 (electronic), 2002.

[4] Mina Aganagic, Vincent Bouchard, and Albrecht Klemm. Topological Strings and (Almost) Modular Forms. arXiv:hep-th/0607100.

[5] Samuel Boissiere and Alessandra Sarti. Contraction of excess fibres between the McKay correspondences in dimensions two and three. Ann. Inst. Fourier, 57(6):1839-1861, 2007. arXiv version: math.AG/0504360.

[6] Vincent Bouchard and Renzo Cavalieri. On the mathematics and physics of high genus invariants of $\left[C^{3} / Z_{3}\right]$. arXiv:math/0709.3805.

[7] Tom Bridgeland, Alastair King, and Miles Reid. The McKay correspondence as an equivalence of derived categories. J. Amer. Math. Soc., 14(3):535-554 (electronic), 2001.

[8] Jim Bryan and Amin Gholampour. Hurwitz-Hodge integrals, the $E_{6}$ and $D_{4}$ root systems, and the Crepant Resolution Conjecture. arXiv:0708.4244.

[9] Jim Bryan and Amin Gholampour. Root systems and the quantum cohomology of ADE resolutions. arXiv:0707.1337.

[10] Jim Bryan and Amin Gholampour. The Quantum McKay correspondence for polyhedral singularities. arXiv:0803.3766.

[11] Jim Bryan, Tom Graber, and Rahul Pandharipande. The orbifold quantum cohomology of $\mathbf{C}^{2} / \mathbf{Z}_{3}$ and Hurwitz Hodge integrals. Journal of Algebraic Geometry, 17:1-28, 2008. arXiv version:math.AG/0510335.

[12] Jim Bryan and Yunfeng Jiang. The Crepant Resolution Conjecture for the orbifold $\mathbf{C}^{2} / \mathbf{Z}_{4}$. In preparation.

[13] Jim Bryan and Rahul Pandharipande. The local Gromov-Witten theory of curves. Journal of the American Mathematical Society, 21:101-136, 2008. arXiv:math.AG/0411037.

[14] Charles Cadman and Renzo Cavalieri. Gerby Localization, $Z_{3}$-Hodge Integrals and the GW Theory of $C^{3} / Z_{3}$. arXiv:math/0705.2158.

[15] Weimin Chen and Yongbin Ruan. Orbifold Gromov-Witten theory. In Orbifolds in mathematics and physics (Madison, WI, 2001), volume 310 of Contemp. Math., pages 25-85. Amer. Math. Soc., Providence, RI, 2002.

[16] Coates. Wall-Crossings in Toric Gromov-Witten Theory II: Local Examples. arXiv:math/0804.2592.

[17] Tom Coates, Alessio Corti, Hiroshi Iritani, and Hsian-Hua Tseng. Computing Genus-Zero Twisted Gromov-Witten Invariants. arXiv:math.AG/0702234.

[18] Tom Coates, Alessio Corti, Hiroshi Iritani, and Hsian-Hua Tseng. The Crepant Resolution Conjecture for Type A Surface Singularities. arXiv:0704.2034v1 [math.AG].

[19] Tom Coates, Alessio Corti, Hiroshi Iritani, and Hsian-Hua Tseng. Wall-Crossings in Toric Gromov-Witten Theory I: Crepant Examples. arXiv:math.AG/0611550.

[20] Tom Coates and Yongbin Ruan. Quantum Cohomology and Crepant Resolutions: A Conjecture. arXiv:0710.5901.

[21] David A. Cox and Sheldon Katz. Mirror symmetry and algebraic geometry. American Mathematical Society, Providence, RI, 1999.

[22] C. Faber and R. Pandharipande. Logarithmic series and Hodge integrals in the tautological ring. Michigan Math. J., 48:215-252, 2000. With an appendix by Don Zagier, Dedicated to William Fulton on the occasion of his 60 th birthday.

[23] Barbara Fantechi and Lothar Göttsche. Orbifold cohomology for global quotients. Duke Math. $J ., 117(2): 197-227,2003$.

[24] Javier Fernandez. Hodge structures for orbifold cohomology. Proc. Amer. Math. Soc., 134:2511-2520, 2006. arXiv:math.AG/0311026.

[25] W. D. Gillam. The Crepant Resolution Conjecture for 3-dimensional flags modulo an involution. arXiv:math/0708.0842. 
[26] T. Graber and R. Pandharipande. Localization of virtual classes. Invent. Math., 135(2):487$518,1999$.

[27] Kentaro Hori, Sheldon Katz, Albrecht Klemm, Rahul Pandharipande, Richard Thomas, Cumrun Vafa, Ravi Vakil, and Eric Zaslow. Mirror symmetry, volume 1 of Clay Mathematics Monographs. American Mathematical Society, Providence, RI, 2003. With a preface by Vafa.

[28] Hiroshi Iritani. Real and integral structures in quantum cohomology I: toric orbifolds. arXiv:math/0712.2204.

[29] Manfred Lehn and Christoph Sorger. Symmetric groups and the cup product on the cohomology of Hilbert schemes. Duke Math. J., 110(2):345-357, 2001.

[30] D. Maulik, N. Nekrasov, A. Okounkov, and R. Pandharipande. Gromov-Witten theory and Donaldson-Thomas theory. I. Compos. Math., 142(5):1263-1285, 2006. arXiv:math.AG/0312059.

[31] Davesh Maulik. Gromov-Witten Theory of A-resolutions, arXiv:0802.2681.

[32] John McKay. Graphs, singularities, and finite groups. In The Santa Cruz Conference on Finite Groups (Univ. California, Santa Cruz, Calif., 1979), volume 37 of Proc. Sympos. Pure Math., pages 183-186. Amer. Math. Soc., Providence, R.I., 1980.

[33] A. Okounkov and R. Pandharipande. The local Donaldson-Thomas theory of curves. arXiv:math.AG/0512573.

[34] Andrei Okounkov and Rahul Pandharipande. Quantum cohomology of the Hilbert scheme of points in the plane. arXiv:math.AG/0411210.

[35] Michael A. Rose. A reconstruction theorem for genus zero Gromov-Witten invariants of stacks. arXiv:math.AG/0605776.

[36] Yongbin Ruan. The cohomology ring of crepant resolutions of orbifolds. In Gromov-Witten theory of spin curves and orbifolds, volume 403 of Contemp. Math., pages 117-126. Amer. Math. Soc., Providence, RI, 2006.

[37] Bernardo Uribe. Orbifold cohomology of the symmetric product. Comm. Anal. Geom., 13(1):113-128, 2005.

[38] Cumrun Vafa. String vacua and orbifoldized LG models. Modern Phys. Lett. A, 4(12):1169$1185,1989$.

[39] Eric Vasserot. Sur l'anneau de cohomologie du schéma de Hilbert de $\mathbf{C}^{2}$. C. R. Acad. Sci. Paris Sér. I Math., 332(1):7-12, 2001.

[40] Jonathan Wise. The genus zero Gromov-Witten invariants of $\left[\mathrm{Sym}^{2} \mathbb{P}^{2}\right]$. arXiv:math/0702219.

[41] Takehiko Yasuda. Twisted jets, motivic measures and orbifold cohomology. Compos. Math., 140(2):396-422, 2004.

[42] Eric Zaslow. Topological orbifold models and quantum cohomology rings. Comm. Math. Phys., 156(2):301-331, 1993.

Dept of Math, Univ. of British Columbia, Vancouver, BC, Canada

E-mail address: jbryan@math.ubc.ca

Dept of Math, California Institute of Technology, Pasadena, CA

E-mail address: graber@caltech.edu 